\title{
Synergistic Activity and Biofilm Formation Effect of Colistin Combined with PFK-I58 Against Colistin-Resistant Gram-Negative Bacteria
}

\author{
Liqiong Chen' \\ Kaihang $\mathrm{Yu}^{2}$ \\ Lijiang Chen' \\ Xiangkuo Zheng' \\ Na Huang' \\ Yishuai $\operatorname{Lin}^{2}$ \\ Huaiyu Jia' \\ Wenli Liao' \\ Jianming $\mathrm{CaO}^{2}$ \\ Tieli Zhou' \\ 'Department of Clinical Laboratory, The \\ First Affiliated Hospital of Wenzhou \\ Medical University, Wenzhou, Zhejiang \\ Province, People's Republic of China; \\ ${ }^{2}$ Department of Medical Laboratory \\ Science, School of Laboratory Medicine \\ and Life Science, Wenzhou Medical \\ University, Wenzhou, Zhejiang Province, \\ People's Republic of China
}

\begin{abstract}
Purpose: The emergence of colistin resistance among Gram-negative bacteria (GNB) poses a serious public health threat. Therefore, it is necessary to enhance the antibacterial activity of colistin through the combination with other drugs. In this study, we demonstrated the synergistic activity and the possible synergy mechanism of colistin with PFK-158 against colistin-resistant GNB, including non-fermenting bacteria and Enterobacteriaceae.
\end{abstract}

Patients and Methods: Thirty-one colistin-resistant GNB, including Pseudomonas aeruginosa $(\mathrm{n}=9)$, Acinetobacter baumannii $(\mathrm{n}=5)$, Escherichia coli $(\mathrm{n}=8)$ and Klebsiella pneumoniae $(\mathrm{n}=9)$, were collected as the experimental strains and the minimum inhibitory concentrations (MICs) of colistin, other routine antimicrobial agents and PFK-158 against all strains were determined by the broth microdilution method. The synergistic activity of colistin with PFK-158 was assessed by the checkerboard assay and time-kill assay. The biofilm formation assay and scanning electron microscopy were used to demonstrate the biofilm formation effect of colistin with PFK-158 against colistin-resistant GNB.

Results: The results of the checkerboard assay showed that when colistin was used in combination with PFK-158, synergistic activity was observed against the 31 colistin-resistant GNB. The time-kill assay presented a significant killing activity of colistin with PFK-158 against the 9 colistin-resistant GNB selected randomly, including Pseudomonas aeruginosa $(\mathrm{n}=6)$, Acinetobacter baumannii $(\mathrm{n}=1)$, Escherichia coli $(\mathrm{n}=1)$, and Klebsiella pneumoniae $(\mathrm{n}=1)$. The biofilm formation assay and scanning electron microscopjihy showed that colistin with PFK-158 can effectively suppress the formation of biofilm and reduce the cell arrangement density of biofilm against most experimental strains.

Conclusion: The results of the performed experiments suggest that the combination of colistin and PFK-158 may be a potential new choice as a new antibiofilm group for the treatment of infections caused by the colistin-resistant GNB.

Keywords: synergy, non-fermenting bacteria, enterobacteriaceae, bacterial biofilm, cytotoxicity

\section{Introduction}

Due to the rapid spread of antimicrobial resistance and the slow development of novel antimicrobials, Gram-negative bacteria (GNB) infections are becoming extremely challenging for clinicians and a real threat to international public health. ${ }^{1,2}$ Rapid spread and dissemination of resistance-encoding genes among pathogens further exacerbate the problem, resulting in an ever-increasing rate of antimicrobialresistant bacterial infections. ${ }^{3-5}$ The traditional drug, colistin, is a defensive drug
Correspondence: Tieli Zhou

Tel +86-577-86689885

Email wyztli@।63.com

Jianming Cao

Tel +86-577-88069595

Email wzcjming@I63.com 
commonly used clinically to treat infections caused by multi-drug resistant and carbapenem-resistant GNB. ${ }^{6}$ Unfortunately, the increased and inappropriate use of colistin has led inexorably to the worldwide emergence of colistin-resistant GNB in the last few years. ${ }^{7-9}$ The emergence of colistin resistance among GNB poses a serious public health threat and warrants immediate action. ${ }^{10-12}$ Therefore, it is necessary to enhance the antibacterial activity of colistin through the combination with other drugs.

Previous studies have shown that infections caused by GNB are often closely related to the formation of biofilm. ${ }^{13,14}$ Bacterial biofilm is the organized bacterial populations formed by extracellular polymers, such as protein, polysaccharides and extracellular DNA. ${ }^{15,16}$ The formation of biofilm can strengthen the ability of bacteria to adapt to the environment and make the penetration of conventional antibacterial drugs difficult; thus, it causes repeated chronic bacterial behavior infection. ${ }^{17-19}$ In addition, the formation of biofilm is also conducive to the spread of drug resistance between strains. ${ }^{20,21}$

PFK-158 is a potent and selective 6-phosphofructo2-kinase/fructose-2, 6-bisphosphatase 3 (PFKFB3) inhibitor, which shows extensive anti-tumor activity by reducing the uptake of glucose in cancer cells, the production of ATP, the release of lactic acid and inducing apoptosis and autophagy. ${ }^{22,23}$ A Phase I clinical trial of PFK-158 was completed in July 2016.

At present, there is only one study having investigated the synergistic activity of colistin combined with PFK-158 against colistin-resistant Enterobacteriaceae. ${ }^{24}$ However, there was no study exploring the synergistic activity of these two drugs against colistin-resistant $P$. aeruginosaand $A$. baumannii. Therefore, the main aim of this study was to determine the synergistic activity and biofilm formation effect of colistin in combination with PFK-158 against colistin-resistant GNB including Pseudomonas aeruginosa ( $P$. aeruginosa $)$ and Acinetobacter baumannii (A. baumannii) and Enterobacteriaceae, in order to provide new possible future therapeutic strategies to combat resistance to this antibiotic of last resort.

\section{Patients and Methods}

\section{Bacterial Strains}

A total of 31 non-duplicated Gram-negative clinical isolates were recovered from the First Affiliated Hospital of
Wenzhou Medical University in China, including colistinresistant $P$. aeruginosa $(\mathrm{n}=9)$, A. baumannii $(\mathrm{n}=5)$, Escherichia coli $($ E. coli $)(\mathrm{n}=8)$, and Klebsiella pneumoniae $($ K. pneumoniae $)(\mathrm{n}=9)$. These isolates were all identified by matrix-assisted laser desorption/ionization time-of-flight mass spectrometry (MALDI-TOF/MS; bioMérieux, Lyons, France). All strains were stored in Luria Bertani (LB) broth medium (Oxoid, Britain) at $-80{ }^{\circ} \mathrm{C}$ containing $30 \% \mathrm{v} / \mathrm{v}$ glycerol for later use. P. aeruginosa ATCC 27,853 and ATCC 25,922 were served as the quality controls, which were purchased from the National Center of Clinical Laboratory (NCCL).

\section{Clinical Data Collection}

The medical records were reviewed to integrally collect the data of the patients with 31 colistin-resistant GNB. The study data included the following variables: gender, age of the patients and isolation date, isolation sample, isolation ward of the strains.

\section{Antimicrobial Agents}

PFK-158 was purchased from MedChemExpress (MCE) Co., Ltd (New Jersey, USA) and dissolved in $2.5 \% \mathrm{v} / \mathrm{v}$ dimethyl sulfoxide (DMSO) (Sigma-Aldrich, Saint Louis, USA). All antibiotics, including colistin, aztreonam, ceftazidime, cefepime, imipenem, ciprofloxacin, levofloxacin, gentamicin, and tobramycin were purchased from Wenzhou Kangtai Biological Technology Co., Ltd (Zhejiang, China).

\section{Antimicrobial Susceptibility Assay}

The MICs of all antibiotics and PFK-158 were determined by the broth microdilution in cation-adjusted Mueller-Hinton Broth (CAMHB). The CAMHB (Biofroxx Biological Technology Co., Ltd., Germany) was prepared by adding an appropriate amount of $\mathrm{Mg}^{2+}$ and $\mathrm{Ca}^{2+}$ into MuellerHinton Broth, with final concentrations of $10.0-12.5 \mathrm{mg} / \mathrm{L}$ and $20-25 \mathrm{mg} / \mathrm{L}$, respectively. The final bacteria concentration of each well was approximately at $7.5 \times 10^{5}$ colony forming units $(\mathrm{CFU}) / \mathrm{mL}$. Serial two-fold dilutions for colistin and PFK-158 were prepared in CAMHB 96-well microtiter plates (Flat bottom with lid, Sterile; Corning, USA). The results were observed after incubation at $37{ }^{\circ} \mathrm{C}$ for $16-18$ h. The MICs of colistin determination for all strains were interpreted by the recommendation of CLSI. Multi-drug resistant (MDR) strains were defined as non-susceptible to three or more different antimicrobial categories. ${ }^{25}$ All experiments were performed in triplicate. 


\section{Checkerboard Assay}

The in-vitro synergistic activity of the combination against the colistin-resistant GNB was examined by using the checkerboard assay on a 96-well plate (Flat bottom with lid, Sterile; Corning, USA) as previously described. Colistin is 2-fold serially diluted along the $\mathrm{x}$-axis, while PFK-158 is 2-fold serially diluted along the $y$-axis to form a matrix. ${ }^{26}$ The final bacteria concentration of each well was also approximately at $7.5 \times 10^{5} \mathrm{CFU} / \mathrm{mL}$. Wells containing CAMHB with or without bacterial cells were used as positive or negative controls, respectively. The 96-well plates were then incubated at $37^{\circ} \mathrm{C}$ for $16-18 \mathrm{~h}$ and record the MICs of the single drug and the $\mathrm{MIC}_{\mathrm{s}}$ of the combination of colistin with PFK-158.

The fractional inhibitory concentration index (FICI) was used to evaluate the potential synergistic activity of the two drugs in combination. The FICI was calculated with the formula: FICI $=$ (MIC of drug A in the combination/MIC of drug A alone) + (MIC of drug $\mathrm{B}$ in the combination/MIC of drug $\mathrm{B}$ alone). $\mathrm{FICI} \leq 0.5$, $0.5<\mathrm{FICI} \leq 1.0,1.0<\mathrm{FICI} \leq 2.0$, FICI $>2.0$ was expected as synergistic activity, additive activity, irrelevant activity, and antagonistic activity, respectively. All experiments were performed in triplicate.

\section{Time-Kill Assay}

The time-kill assay was conducted according to a published protocol with several modifications. ${ }^{27,28}$ In brief, we randomly selected 9 strains as experimental strains, including colistin-resistant $P$. aeruginosa $(\mathrm{n}=6)$, A. baumannii $(\mathrm{n}=1), E$. coli $(\mathrm{n}=1)$, and $K$. pneumoniae $(\mathrm{n}=1)$. The synergistic activity of these two drugs was achieved with combinations of colistin and PFK-158 used at the lowest concentration when it showed synergistic activity. At the same time, we set up the control group adding colistin and PFK-158 alone. Viable cells were counted by spreading $100 \mu \mathrm{L}$ of samples after appropriate dilutions with saline and plating on antibiotic-free Mueller-Hinton agar plates at $0,2,4,6,12$, and 24 $\mathrm{h}$ after antibiotic addition. Bacterial colonies were counted from the plates after incubation for $16-18 \mathrm{~h}$ at $37^{\circ} \mathrm{C}$. The assay was performed in triplicate for these strains; then, we calculated the average value of viable CFU and plotted it on a semi-logarithmic graph. Antimicrobial carryover was controlled by the inhibition of colonial growth at the site of the initial streak according to NCCLS guidelines. ${ }^{27}$ Bactericidal activity was defined as $\mathrm{a} \geq 3 \log 10$ decrease in
CFU/mL by $24 \mathrm{~h}$. Synergistic activity was defined as a $\geq 2$ $\log 10$ decrease in CFU/mL at $24 \mathrm{~h}$ by the two drugs combination compared with the single drug. ${ }^{27}$

\section{Biofilm Formation and Eradication Assay}

The biofilm biomass was detected by crystal violet staining as previously described. ${ }^{29}$ As for the biofilm formation assay, after inoculation for $18 \mathrm{~h}$, strains were diluted 1:100 in fresh LB broth. $100 \mu \mathrm{L} \mathrm{LB}$ broth and an aliquot of each sample was then transferred to a 96-well microtiter plate (Flat bottom with lid, Sterile; Corning, USA) and incubated at $37{ }^{\circ} \mathrm{C}$ for $24 \mathrm{~h}$. The biofilm was stained with 150 $\mu \mathrm{L} 1 \% \mathrm{w} / \mathrm{v}$ crystal violet staining solution (lot number: NO.20190324, Beijing Solarbio Biotechnology Co., Ltd., China) for 15 minutes at $37^{\circ} \mathrm{C}$, and each well was washed twice with normal saline. Add $150 \mu \mathrm{L}$ of $95 \% \mathrm{v} / \mathrm{v}$ ethanol (95\% absolute ethanol and 5\% glacial acetic acid) for 10 minutes at $37{ }^{\circ} \mathrm{C}$ to solubilized the stained biofilms, and the $\mathrm{OD}_{595}$ were measured with a microplate reader (Multiskan FC). The difference between the biofilm formation and eradication experiment step was that the former added the drug before the biofilm was formed, and the latter added the drug after the mature biofilm was formed.

\section{Scanning Electron Microscope}

The biofilm of TL2314 was treated with or without colistin (at $2 \mu \mathrm{g} / \mathrm{mL}$ ), PFK-158 (at the lowest concentrations that can show synergistic effects when combined with colistin) alone and in combination for $24 \mathrm{~h}$ as described above on sterile cover slips (lot number: NO.10211818C, CITOGLAS Co., Ltd., China) in a 6-well plate (NEST Biotechnology Co., Ltd., China).$^{30}$ After treatments, the cover slips were rinsed by sterile PBS for three times, and then fixed by $2.5 \%(\mathrm{v} / \mathrm{v})$ glutaraldehyde overnight. After drying, the samples were dehydrated by increasing concentrations of ethanol $(20 \%$, $40 \%, 70 \%, 90 \%, 95 \%$ and $100 \%$, v/v, 2 min each). Finally, the samples were processed by gold sputtering and observed by SEM (S-3000N, Japan).

\section{Cytotoxicity Assay by MTT Method}

RAW 264.7 macrophage cell lines were purchased commercially from Procell Life Science and Technology Co., Ltd., China. Cytotoxicity of PFK-158 with different concentrations $(1,2,4,8,16,32,64$, and $128 \mu \mathrm{g} / \mathrm{mL})$ and $2.5 \%$ DMSO was performed with RAW 264.7 macrophage cell lines with minor modifications. $2 \times 10^{5}$ macrophage cell lines were seeded into each well of 96-well tissue culture plates (Flat bottom with lid, Sterile; Corning, USA) and 
cultured in Roswell Park Memorial Institute (RPMI-1640) supplemented with $10 \% \mathrm{v} / \mathrm{v}$ fetal calf serum (FCS). And then cell lines were treated with PFK-158, 2.5\% v/v DMSO and incubated in the presence of $5 \% \mathrm{v} / \mathrm{v} \mathrm{CO}_{2}$ at $37^{\circ} \mathrm{C}$ for 24 $\mathrm{h}$. After that, the medium was replaced with a fresh RPMI containing $5 \mathrm{mg} / \mathrm{mL} \quad 3$-(4, 5-Dimethylthiazol-2-yl)2,5-diphenyltetrazolium bromide (MTT) and incubated at $37{ }^{\circ} \mathrm{C}$ for $4 \mathrm{~h}$. Followed by that, formazan crystals were dissolved in DMSO by 10 min incubation at room temperature and absorbance was measured using a microplate reader (SpectraMax 190) at test wavelength of " $\lambda "=490 \mathrm{~nm}^{31}$

\section{Statistical Analysis}

Each experiment was performed in triplicate. Significance was determined by using two-sample $t$-test and mentioned as $P$ value $<0.01$ (noted with*), $P$ value $<0.001$ (noted with ${ }^{* *}$ ) and $P$ value $<0.0001$ (noted with***). Statistical analyses were performed using Graph Pad Prism 7.03 statistical software.

\section{Results}

\section{Bacterial Strains and Antimicrobial Agents and Susceptibility Test}

Table 1 summarizes the patients characteristics and species distribution. Overall, the colistin-resistant $P$. aeruginosa strains were mainly from the sputum sample $(88.9 \%, 8 / 9)$. The colistin-resistant A. baumannii strains were all from the sputum sample $(100.0 \%, 5 / 5)$. Most of the colistin-resistant K. pneumoniae strains were

Table I Patient's Clinical Data and Characteristics of Analyzed Strains

\begin{tabular}{|c|c|c|c|c|c|c|}
\hline Species & Strains & Isolation Date & Age & Gender & Sample & Ward \\
\hline \multirow[t]{9}{*}{ P. aeruginosa } & TLI67I & $11 / 04 / 2015$ & 74 & $M$ & Wound & Endocrinology \\
\hline & TLI722 & $16 / 05 / 2015$ & 63 & $M$ & Sputum & Neurosurgery \\
\hline & TLI736 & $20 / 05 / 2015$ & 44 & $M$ & Sputum & Neurosurgery \\
\hline & TLI744 & $23 / 05 / 2015$ & 63 & $M$ & Sputum & $\mathrm{ICU}$ \\
\hline & TL23|4 & $08 / 03 / 2016$ & 66 & $M$ & Sputum & ICU \\
\hline & TL29I7 & $09 / 02 / 2017$ & 58 & $M$ & Sputum & ICU \\
\hline & TL2967 & $13 / 03 / 20 \mid 7$ & 26 & $M$ & Sputum & Emergency \\
\hline & TL3008 & $05 / 04 / 2017$ & 67 & $M$ & Sputum & Neurosurgery \\
\hline & TL3086 & $24 / 05 / 2017$ & 65 & $M$ & Sputum & Neurosurgery \\
\hline \multirow[t]{5}{*}{ A. baumannii } & BM2370 & $25 / 02 / 2015$ & 59 & $M$ & Sputum & ICU \\
\hline & BM243I & $19 / 03 / 2015$ & 89 & $M$ & Sputum & ICU \\
\hline & BMI539 & $\mid \mathrm{I} / 02 / 2014$ & 47 & $M$ & Sputum & ICU \\
\hline & BM24I2 & $13 / 03 / 20 \mid 5$ & 63 & $\mathrm{~F}$ & Sputum & $\mathrm{ICU}$ \\
\hline & BMI595 & $28 / 02 / 2014$ & 57 & $F$ & Sputum & Neurosurgery \\
\hline \multirow[t]{8}{*}{ E. coli } & DC90 & $27 / 03 / 2012$ & 76 & $M$ & Wound & Gastrointestinal Surgery \\
\hline & DC34II & $26 / 02 / 2015$ & 75 & M & Urine & Urology \\
\hline & DC3539 & $26 / 03 / 2015$ & 82 & M & Drainage & Gastrointestinal Surgery \\
\hline & DC3599 & $07 / 04 / 2015$ & 72 & $M$ & Sputum & Respiratory Medicine \\
\hline & DC3737 & $05 / 05 / 2015$ & 52 & $M$ & Wound & Orthopedics \\
\hline & DC3806 & $19 / 05 / 2015$ & 17 & $\mathrm{~F}$ & Sputum & Hematology \\
\hline & DC4887 & $23 / 02 / 2016$ & 63 & $M$ & Urine & Urology \\
\hline & DC5262 & $18 / 05 / 20 \mid 6$ & 32 & $\mathrm{~F}$ & Urine & Emergency \\
\hline \multirow[t]{9}{*}{ K. pneumoniae } & FKI342 & $04 / 06 / 2014$ & 21 & $\mathrm{~F}$ & Sputum & Anorectal Surgery \\
\hline & FK26 & $15 / 03 / 2012$ & 65 & M & Sputum & Neurosurgery \\
\hline & FK6556 & $04 / 04 / 2019$ & 41 & $\mathrm{~F}$ & Sputum & Respiratory Medicine \\
\hline & FK20 & $08 / 03 / 2012$ & 88 & $\mathrm{~F}$ & Sputum & Neurology \\
\hline & FKI913 & $19 / 0|/ 20| 5$ & 74 & $M$ & Sputum & Neurosurgery \\
\hline & FK610 & $13 / 05 / 2013$ & 83 & M & Sputum & Trauma surgery \\
\hline & FK59I & $02 / 05 / 2013$ & 65 & $\mathrm{~F}$ & Urine & Neurology \\
\hline & FKI69 & $02 / 07 / 2012$ & 79 & $F$ & Sputum & General \\
\hline & FKI50 & $19 / 06 / 2012$ & 60 & $M$ & Pus & Emergency \\
\hline
\end{tabular}

Abbreviations: M, male; F, female. 
from the sputum sample $(77.8 \%, 7 / 9)$. But the separation site of colistin-resistant E. coli was relatively scattered, and the proportion of urine, sputum, wound and drainage were $37.5 \%$ (3/8), 25.0\% (2/8), 25.0\% (2/8), 12.5\% (1/8), respectively. There were more strains from males than females $(71.0 \%$ vs $29.0 \%$, respectively). Strains were cultured from patients aged 17 to 89 years (average age 60.8 years). The colistin-resistant $P$. aeruginosa strains were mainly from patients in neurosurgery $(44.4 \%, 4 / 9)$, the intensive care unit (ICU) $(33.3 \%, 3 / 9)$. Most of the colistin-resistant A. baumannii strains were from patients in the intensive care unit (ICU) $(80.0 \%, 4 / 5)$. However, the isolation wards of the remaining two strain species were relatively scattered.

As shown in Table 2, all the 31 GNB were all resistant to colistin $(>2 \mu \mathrm{g} / \mathrm{mL})$. In addition, MICs of PFK-158 were $512 \mu \mathrm{g} / \mathrm{mL}$ for all tested strains, showing the lack of any intrinsic antimicrobial activity of PFK-158 alone against these Gram-negative strains. And all of the colistin-resistant $A$. baumannii $(\mathrm{n}=5)$, E. coli $(\mathrm{n}=8)$, $K$. pneumoniae $(\mathrm{n}=9)$ and 4 of 9 colistin-resistant $P$. aeruginosa exhibited multidrug-resistance.

Table 2 Representative MICs Against 3I Colistin-Resistant GNB

\begin{tabular}{|c|c|c|c|c|c|c|c|c|c|c|c|c|}
\hline \multirow[t]{2}{*}{ Species } & \multirow[t]{2}{*}{ Strains } & Antibiotics & ATM & CAZ & FEP & IPM & CIP & LVX & GEN & ТОВ & COL & PFK-I 58 \\
\hline & & Breakpoints(S-R) & $8-32$ & $8-32$ & $8-32$ & $2-8$ & $0.5-2$ & $\mathrm{I}-4$ & $4-16$ & $4-16$ & $2-4$ & \\
\hline \multirow[t]{9}{*}{$P$. aeruginosa } & TLI67| & MICs ( $\mu \mathrm{g} / \mathrm{mL})$ & 8 & 4 & 8 & 2 & 0.25 & I & 2 & 1 & 8 & 512 \\
\hline & TLI722 & & 32 & 64 & 16 & 16 & 4 & 4 & 32 & 4 & 8 & 512 \\
\hline & TLI736 & & 4 & 4 & 2 & 16 & I & I & 32 & 8 & 16 & 512 \\
\hline & TLI 744 & & 32 & 32 & 16 & 16 & 32 & 8 & $\geq \mathbf{1 2 8}$ & 32 & 8 & 512 \\
\hline & TL23I4 & & 16 & 32 & 16 & 4 & 0.5 & 2 & 8 & 2 & 8 & 512 \\
\hline & TL2917 & & 32 & 16 & 16 & 16 & 0.25 & 2 & 8 & 8 & 16 & 512 \\
\hline & TL2967 & & 128 & 16 & 32 & 16 & 8 & 16 & 8 & 8 & 8 & 512 \\
\hline & TL3008 & & 4 & 2 & 4 & 16 & 0.5 & 1 & 16 & 4 & 32 & 512 \\
\hline & TL3086 & & 128 & 16 & 16 & $\geq 128$ & 16 & 8 & $\geq \mathbf{1 2 8}$ & 128 & 32 & 512 \\
\hline \multirow[t]{5}{*}{ A. baumannii } & BM2370 & MICs $(\mu \mathrm{g} / \mathrm{mL})$ & 8 & 32 & 128 & 8 & 128 & 8 & 4 & I & 16 & 512 \\
\hline & BM243 I & & 64 & 64 & 64 & 16 & 4 & 8 & I & 1 & 32 & 512 \\
\hline & BMI539 & & 16 & 8 & 64 & 16 & 4 & 2 & I & 1 & 16 & 512 \\
\hline & BM24I 2 & & 16 & 64 & 64 & 16 & 4 & 8 & 4 & I & 8 & 512 \\
\hline & BMI595 & & 2 & 32 & 8 & 4 & 64 & 8 & $\geq 128$ & $\geq 128$ & 4 & 512 \\
\hline \multirow[t]{8}{*}{ E. coli } & DC90 & MICs $(\mu \mathrm{g} / \mathrm{mL})$ & $\geq \mathbf{1 2 8}$ & 32 & 64 & $\geq \mathbf{1 2 8}$ & 64 & 32 & $\geq \mathbf{1 2 8}$ & 128 & 8 & 512 \\
\hline & DC34II & & 64 & 32 & 16 & 1 & 64 & 32 & 4 & 2 & 8 & 512 \\
\hline & DC3539 & & 64 & 128 & $\geq \mathbf{1 2 8}$ & 0.5 & 64 & 16 & 128 & 128 & 32 & 512 \\
\hline & DC3599 & & 64 & 16 & 8 & I & 4 & 8 & 16 & 8 & 4 & 512 \\
\hline & DC3737 & & 64 & 64 & 64 & 16 & 4 & 8 & 16 & 16 & 4 & 512 \\
\hline & DC3806 & & 64 & 64 & 16 & I & 4 & 8 & 16 & 16 & 4 & 512 \\
\hline & DC4887 & & I & 4 & 32 & 1 & 4 & 16 & 16 & 8 & 8 & 512 \\
\hline & DC5262 & & $\geq \mathbf{1 2 8}$ & 16 & 16 & 0.25 & $\geq 128$ & 128 & 32 & 16 & 32 & 512 \\
\hline \multirow[t]{9}{*}{ K. pneumoniae } & FK 1342 & MICs $(\mu \mathrm{g} / \mathrm{mL})$ & 128 & $\geq \mathbf{1 2 8}$ & $\geq \mathbf{1 2 8}$ & 0.25 & I & 0.5 & I & 4 & $\geq 128$ & 512 \\
\hline & FK26 & & $\geq 128$ & 64 & 16 & 0.5 & 2 & 0.5 & $\geq 128$ & $\geq 128$ & $\geq 128$ & 512 \\
\hline & FK6556 & & 64 & 64 & 64 & 16 & 4 & 8 & 16 & 16 & 32 & 512 \\
\hline & FK20 & & $\geq \mathbf{1 2 8}$ & $\geq \mathbf{1 2 8}$ & 64 & 0.25 & I & 2 & $\geq \mathbf{1 2 8}$ & $\geq 128$ & $\geq 64$ & 512 \\
\hline & FK1913 & & $\geq \mathbf{1 2 8}$ & $\geq \mathbf{1 2 8}$ & $\geq 128$ & 32 & $\geq 128$ & 128 & $\geq \mathbf{1 2 8}$ & $\geq 128$ & 64 & 512 \\
\hline & FK610 & & $\geq \mathbf{1 2 8}$ & $\geq \mathbf{1 2 8}$ & $\geq \mathbf{1 2 8}$ & 32 & 128 & 32 & 128 & 64 & 8 & 512 \\
\hline & FK59 I & & $\geq \mathbf{1 2 8}$ & $\geq \mathbf{1 2 8}$ & 64 & 0.12 & $\geq 128$ & 128 & I & 8 & 32 & 512 \\
\hline & FKI69 & & $\geq 128$ & 32 & 32 & 0.25 & 32 & 16 & 2 & 2 & 8 & 512 \\
\hline & FKI50 & & $\geq 128$ & 64 & 32 & 0.12 & $\geq 128$ & 32 & 16 & 4 & 16 & 512 \\
\hline
\end{tabular}

Notes: Bolded MICs values mean resistance. Bolded strain number indicates multidrug resistant (MDR) strain. S-R represents the susceptible (S) breakpoint to resistant (R) breakpoint, according to CLSI supplement MI00 (30th edition) and EUCAST.

Abbreviations: GNB, Gram-negative bacteria; ATM, aztreonam; CAZ, ceftazidime; FEP, cefepime; IMP, imipenem; CIP, ciprofloxacin; LVX, levofloxacin; GEN, gentamicin; TOB, tobramycin, COL, colistin. 


\section{Synergistic Activity Testing by the}

\section{Checkerboard Assay}

The checkerboard assay demonstrated that the FICIs of all 31 colistin-resistant GNB were $<0.5$ (Table 3), which included $P$. aeruginosa $(\mathrm{n}=9)$, A. baumannii $(\mathrm{n}=5)$, E. coli $(\mathrm{n}=8)$, and $K$. pneumoniae $(\mathrm{n}=9)$, indicating significant synergistic activity of colistin combined with PFK-158 against colistin-resistant GNB. We observed that the combination of colistin and PFK-158 could reduce colistin MICs of the colistin-resistant strains by 4 to 32 fold. Therefore, the combination of colistin and PFK-158 was selected for further investigation.

\section{Time-Kill Assay and Strains Activity}

Time-kill curves against 9 colistin-resistant strains TL1671，TL1736，TL1744，TL2314，TL3008，TL3086,

Table 3 Summary of MICs and FICl of Colistin Combined with PFK-I58 Against 3I Colistin-Resistant GNB and 2 Quality Control Strains

\begin{tabular}{|c|c|c|c|c|c|c|c|}
\hline \multirow[t]{2}{*}{ Colistin-Resistant GNB } & \multirow[t]{2}{*}{ Strains } & \multicolumn{2}{|c|}{$\begin{array}{l}\text { Antimicrobial Susceptibility } \\
\text { (MIC, } \mu \mathrm{g} / \mathrm{mL})\end{array}$} & \multicolumn{4}{|c|}{ Antimicrobial Combination (MIC, $\mu \mathrm{g} / \mathrm{mL}$ ) } \\
\hline & & COL & PFK-I58 & COL + PFK-I 58 & $\mathbf{F I C l}$ & Potentiation & Interpretation \\
\hline \multirow[t]{10}{*}{ P. aeruginosa } & TLI67। & 8 & 512 & $1+16$ & 0.16 & 8-fold & Synergy \\
\hline & TLI722 & 8 & 512 & $2+16$ & 0.28 & 4-fold & Synergy \\
\hline & TLI736 & 16 & 512 & $4+16$ & 0.28 & 4-fold & Synergy \\
\hline & TLI744 & 8 & 512 & $2+16$ & 0.28 & 4-fold & Synergy \\
\hline & TL23I4 & 8 & 512 & $2+16$ & 0.28 & 4-fold & Synergy \\
\hline & TL2917 & 16 & 512 & $4+32$ & 0.31 & 4-fold & Synergy \\
\hline & TL2967 & 8 & 512 & $2+32$ & 0.31 & 4-fold & Synergy \\
\hline & TL3008 & 32 & 512 & $8+16$ & 0.28 & 4-fold & Synergy \\
\hline & TL3086 & 32 & 512 & $8+32$ & 0.31 & 4-fold & Synergy \\
\hline & ATCC 27,853 & I & 512 & $0.5+16$ & 0.53 & 2-fold & Additivity \\
\hline \multirow[t]{5}{*}{ A. baumannii } & BM2370 & 16 & 512 & $1+8$ & 0.08 & 16-fold & Synergy \\
\hline & BM243I & 32 & 512 & $4+16$ & 0.16 & 8-fold & Synergy \\
\hline & BMI539 & 16 & 512 & $4+32$ & 0.31 & 4-fold & Synergy \\
\hline & BM24I 2 & 8 & 512 & $1+8$ & 0.14 & 8 -fold & Synergy \\
\hline & BMI595 & 4 & 512 & $1+8$ & 0.27 & 4-fold & Synergy \\
\hline \multirow[t]{9}{*}{ E. coli } & DC90 & 8 & 512 & $2+16$ & 0.28 & 4-fold & Synergy \\
\hline & DC34II & 8 & 512 & $1+32$ & 0.19 & 8 -fold & Synergy \\
\hline & DC3539 & 32 & 512 & $4+16$ & 0.16 & 8 -fold & Synergy \\
\hline & DC3599 & 4 & 512 & $1+8$ & 0.27 & 4-fold & Synergy \\
\hline & DC3737 & 4 & 512 & $1+8$ & 0.27 & 4-fold & Synergy \\
\hline & DC3806 & 4 & 512 & $1+16$ & 0.28 & 4-fold & Synergy \\
\hline & DC4887 & 8 & 512 & $1+8$ & 0.14 & 8-fold & Synergy \\
\hline & DC5262 & 32 & 512 & $4+8$ & 0.14 & 8-fold & Synergy \\
\hline & ATCC 25,922 & 0.5 & 512 & $0.25+16$ & 0.53 & 2 -fold & Additivity \\
\hline \multirow[t]{9}{*}{ K. pneumoniae } & FKI 342 & $\geq 128$ & 512 & $8+32$ & 0.13 & 16-fold & Synergy \\
\hline & FK26 & $\geq 128$ & 512 & $16+16$ & 0.16 & 8-fold & Synergy \\
\hline & FK6556 & 32 & 512 & $4+32$ & 0.19 & 8 -fold & Synergy \\
\hline & FK20 & $\geq 64$ & 512 & $2+4$ & 0.04 & 32 -fold & Synergy \\
\hline & FK1913 & 64 & 512 & $2+4$ & 0.04 & 32-fold & Synergy \\
\hline & FK610 & 8 & 512 & $1+4$ & 0.13 & 8-fold & Synergy \\
\hline & FK59I & 32 & 512 & $4+4$ & 0.13 & 8-fold & Synergy \\
\hline & FKI69 & 8 & 512 & $1+4$ & 0.13 & 8 -fold & Synergy \\
\hline & FKI50 & 16 & 512 & $1+16$ & 0.09 & 16-fold & Synergy \\
\hline
\end{tabular}

Notes: Colistin MIC breakpoint for above strains: susceptible, $\leq 2 \mu \mathrm{g} / \mathrm{mL}$; resistant, $>2 \mu \mathrm{g} / \mathrm{mL}$.

Abbreviations: GNB, Gram-negative bacteria; MIC, minimum inhibitory concentration; COL, colistin; FICl, fractional inhibitory concentrations index. 
BM2431, DC3539, and FK1342 are revealed in Figure 1. With colistin or PFK-158 alone, all colistin-resistant strains showed a regrowth after a reduction at $2 \mathrm{~h}$. The combination of colistin and PFK-158 exhibited constant both synergistic and bactericidal activities for TL1736, TL2314, TL3008, TL3086, BM2431, DC3539, and FK1342 for $24 \mathrm{~h}$. As for TL1671, synergistic activity was observed over $24 \mathrm{~h}$ for the combination of colistin and PFK-158, compared with colistin or PFK-158 alone. The combination showed a synergistic activity at 6 $\mathrm{h}$ against TL1744, but regrowth was observed at $24 \mathrm{~h}$. In summary, this combination of colistin and PFK-158 enhanced their killing activity of drug exposure.

\section{Influence of Colistin Combined with PFK-I58 on Biofilm Formation and Eradication}

Biofilm formation and eradication were determined using a crystal violet assay. Figure 2 shows that the combination of colistin and PFK-158 $(p<0.05)$ were found to effectively suppress the formation of biofilm on all the colistin-resistant
P. aeruginosa $(\mathrm{n}=9)$, A. baumannii $(\mathrm{n}=5), K$. pneumoniae $(\mathrm{n}$ $=9)$ and 4 of 8 colistin-resistant $E$. coli compared with the group of using colistin or PFK-158 alone and the control group as for 24-h preformed biofilm. However, as shown in Figure 3, the combination of colistin and PFK-158 had no obvious eradication effect on mature biofilm of all the experimental strain, compared to the control group $(p>0.05)$.

\section{Images of Scanning Electron Microscopy}

In scanning electron microscopy (SEM), the biofilm matrixes of TL2314 were almost removed and the biofilm cells were decreased significantly when using colistin in combination with PFK-158 compared with the drug-free control and colistin alone (Figure 4).

\section{Evaluation of in-vitro Cytotoxicity of PFK-I58 and DMSO}

Figure 5 indicates that the PFK-158 $(<32 \mu \mathrm{g} / \mathrm{mL})$ and $2.5 \%$ dimethyl sulfoxide (DMSO) exerted negligible cytotoxicity on the RAW 264.7 macrophage cell lines compared with the control group treated with MTT $(P>0.05)$.
A

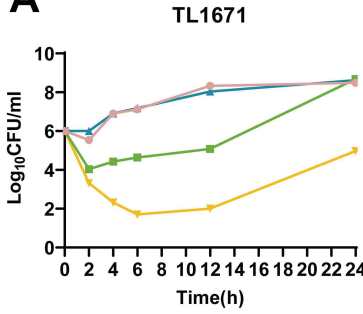

D $\quad$ TL2314
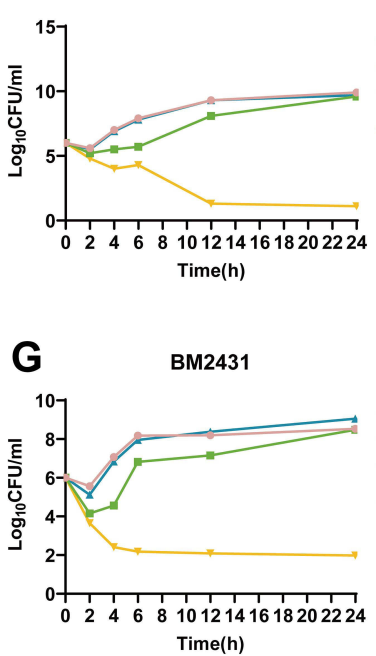

B
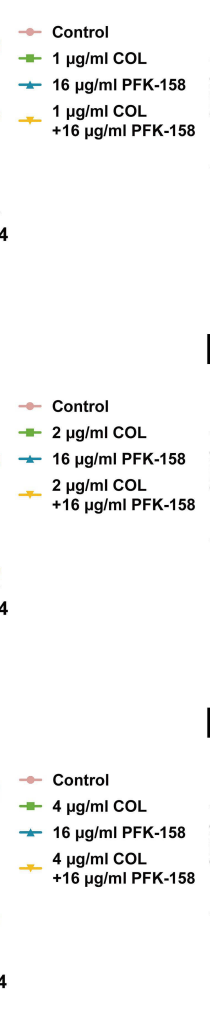

E

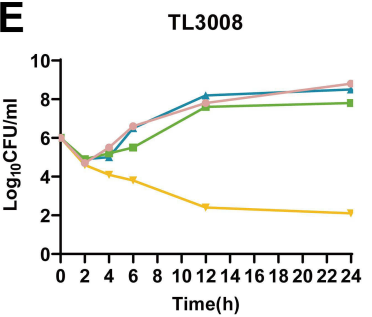

B $\quad$ TL1736
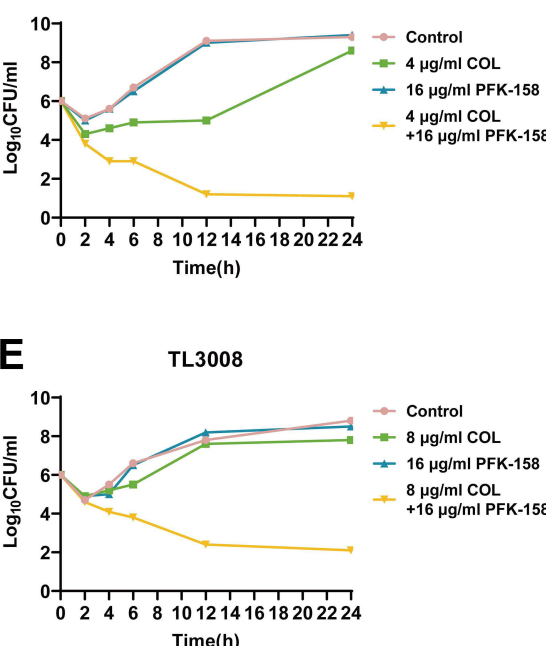

H DC3539
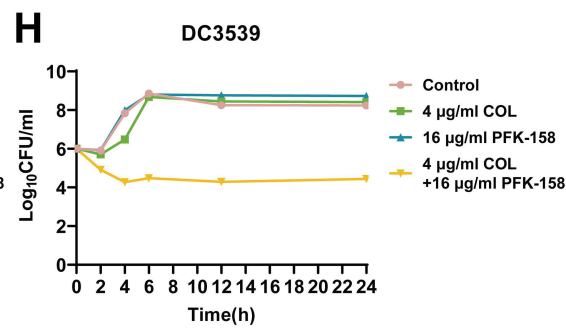

C TL1744

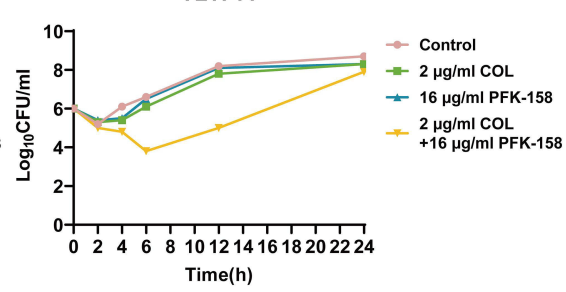

$\mathbf{F}$

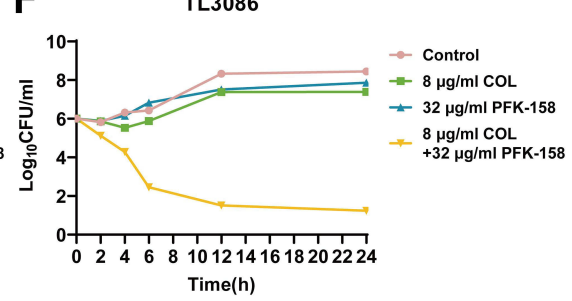

I

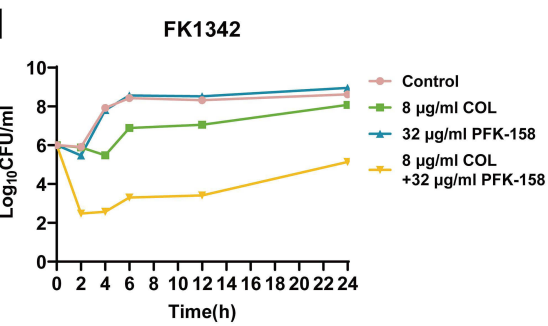

Figure I Time-killing curves of colistin and PFK-I58 alone or in combination against 9 colistin-resistant GNB. (A) TLI67I, (B) TLI736, (C) TLI744, (D) TL23I4, (E) TL3008, (F) TL3086, (G) BM243I, (H) DC3539, (I) FKI342.

Abbreviation: GNB, Gram-negative bacteria. 


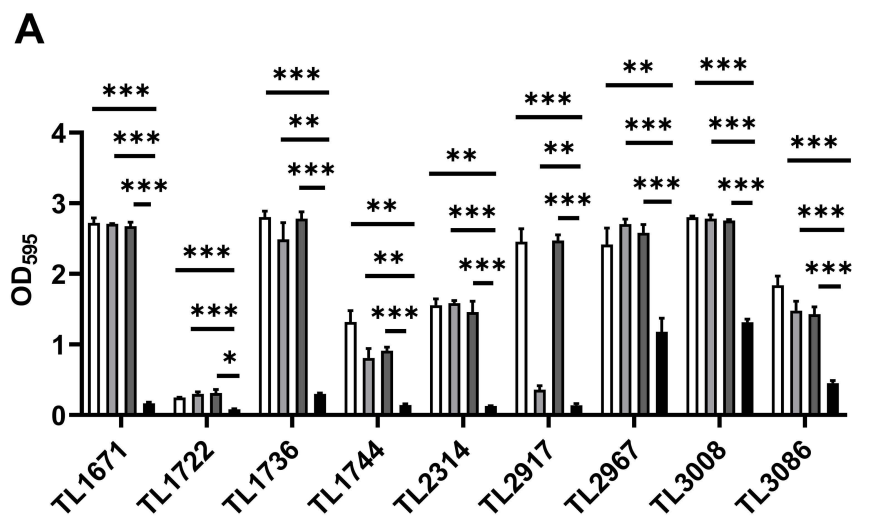

B

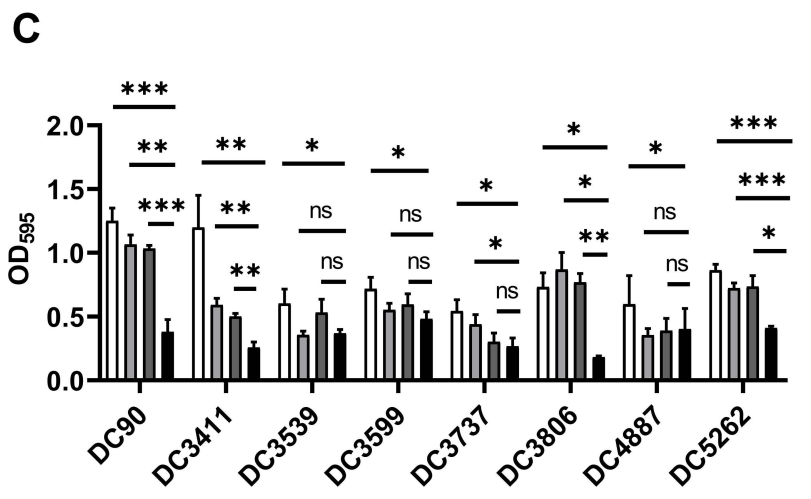

Figure 2 Biofilm inhibitory effects of colistin combined with PFK-I58 on colistin-resistant GNB. (A) P. aeruginosa; (B) A. baumannii; (C) E. coli; (D) K. pneumoniae. *p < 0.05 ; $*_{*}^{*} p<0.01$; *** $p<0.001$. (determined by a two-sample $t$-test). The results are shown as the mean and standard deviation of three independent experiments. Abbreviations: ns, not significant; GNB, Gram-negative bacteria.

\section{Discussion}

The current epidemiological situation is especially worrying with multidrug-resistant (MDR) GNB spreading worldwide and with a paucity of novel marketed antibiotics; thus, colistin is gaining increasing interest from many clinicians worldwide. ${ }^{32}$ However, the increasing numbers of discoveries of colistin-resistant pathogens broke the last barrier between super-bacteria and humans, and new therapy strategies are urgently required. ${ }^{33}$ A number of previous studies have reported the synergistic activity of colistin combined with other antibiotics against colistin-resistant or carbapenem-resistant strains. ${ }^{34-37}$ Besides, a few studies based on colistin in combination with the nonantibiotics were also performed previously. ${ }^{38-40}$ In this study, we evaluated the synergistic activity and biofilm formation effect of colistin combined with PFK-158 against colistin-resistant GNB, including non-fermenting bacteria ( $P$. aeruginosa, A. baumannii) and Enterobacteriaceae (E. coli and K. pneumoniae).

Firstly, as shown in Table 2, it demonstrated that all the colistin-resistant A. baumannii $(\mathrm{n}=5)$, E. coli $(\mathrm{n}=8)$,
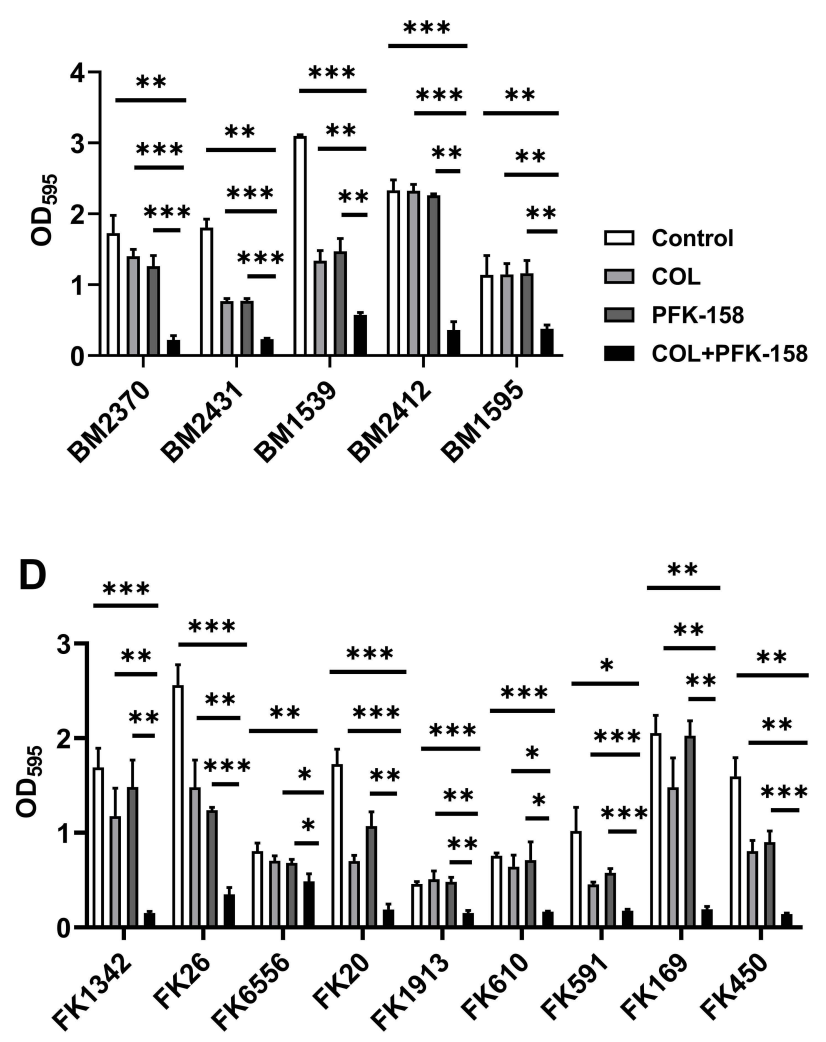

K. pneumoniae $(\mathrm{n}=9)$ and 4 of 9 colistin-resistant $P$. aeruginosa exhibited multi-drug resistance. In other words, the proportion of multidrug-resistant strains in colistin-resistant GNB was relatively high in this study. Therefore, finding a potential new drug group for the treatment of infections caused by the colistin-resistant GNB seems particularly important.

Secondly, as shown in Table 3, we noticed that for all the 31 colistin-resistant GNB we tested, the addition of PFK-158 demonstrated the enhanced colistin susceptibility by reducing colistin MICs (4-32 fold) and fractional inhibitory concentration indices $\left(\mathrm{FICI}_{\mathrm{S}}\right)$ of the combination of colistin and PFK-158 were all below 0.5, indicating that there was a synergistic activity of colistin combined with PFK-158. It should be emphasized that a synergistic activity was observed against all tested colistin-resistant P. aeruginosa, A. baumannii, E.coli, and K. pneumoniae, suggesting that the combination may be a potential strategy to treat the infections caused by these four kinds of colistin-resistant GNB. Recently, Zhang et al have reported on the synergistic activity of colistin combined 
A

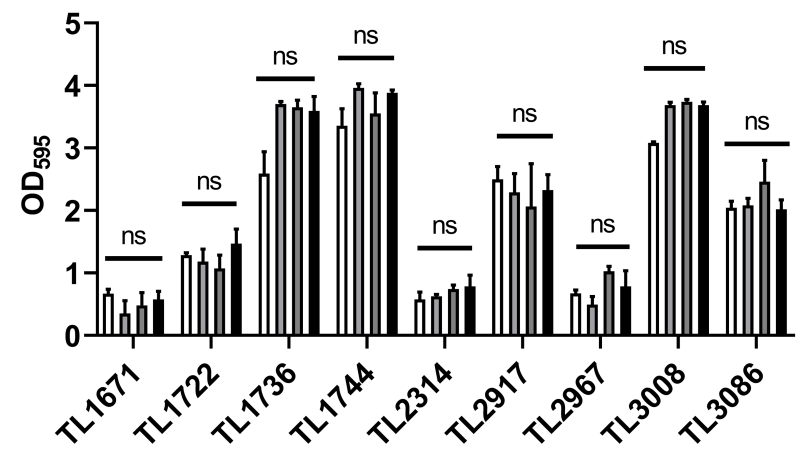

C

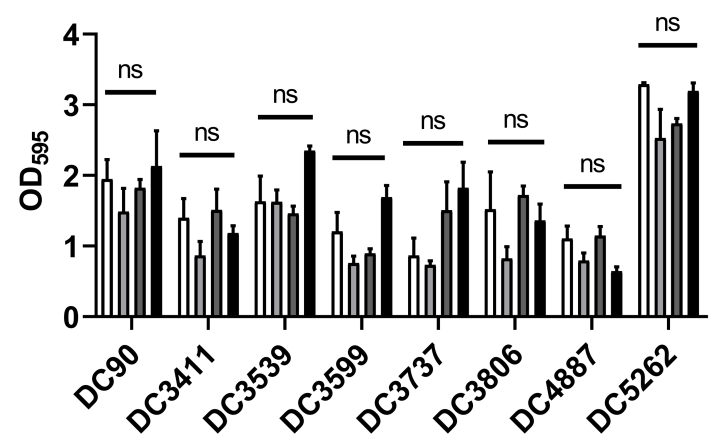

B

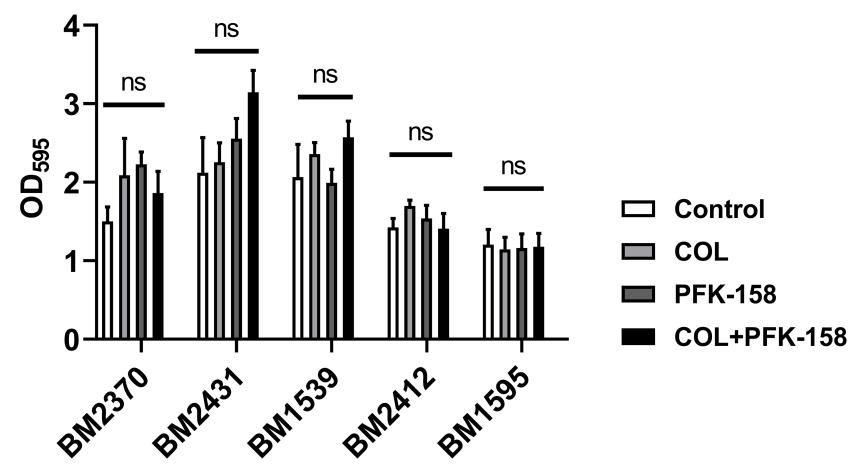

D

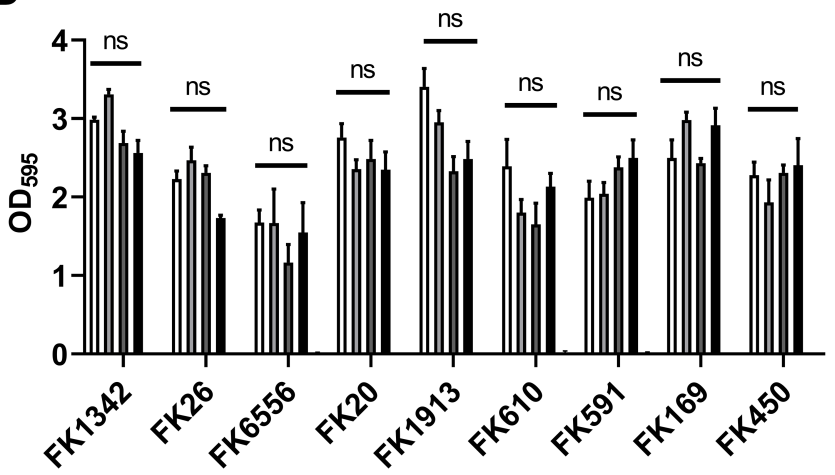

Figure 3 Biofilm eradication effects of colistin combined with PFK-I58 on colistin-resistant GNB. (A) P. aeruginosa; (B) A. baumannii; (C) E. coli; (D) K. pneumoniae. (determined by a two-sample $t$-test). The results are shown as the mean and standard deviation of three independent experiments.

Abbreviations: ns, not significant; GNB, Gram-negative bacteria.

\section{TL2314}
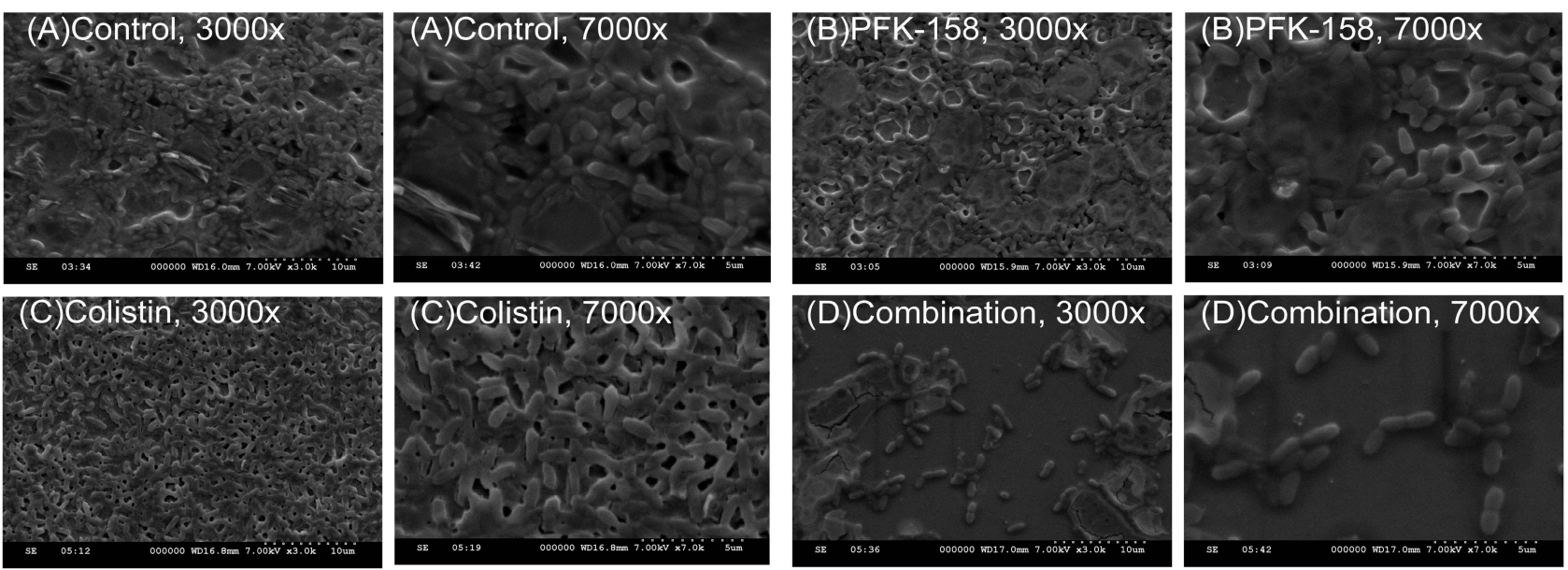

Figure 4 Images from SEM for colistin-resistant P. aeruginosa TL23I 4 exposed to different condition. (A) Control; (B) I6 $\mu$ g/mL PFK-I58 alone; (C) $2 \mu g / \mathrm{mL}$ colistin alone; and (D) $2 \mu \mathrm{g} / \mathrm{mL}$ colistin and $16 \mu \mathrm{g} / \mathrm{mL}$ PFK-I58 in combination.

Abbreviation: SEM, scanning electron microscopy.

with $\quad$ PFK-158 against colistin-resistant Enterobacteriaceae ${ }^{24}$ including E.coli, K. pneumoniae, and E. cloacae, the result of which is consistent with the result in our study. Compared to the study of Zhang et al, the advantage of our study is that we have explored not only the synergistic activity of colistin in combination with 


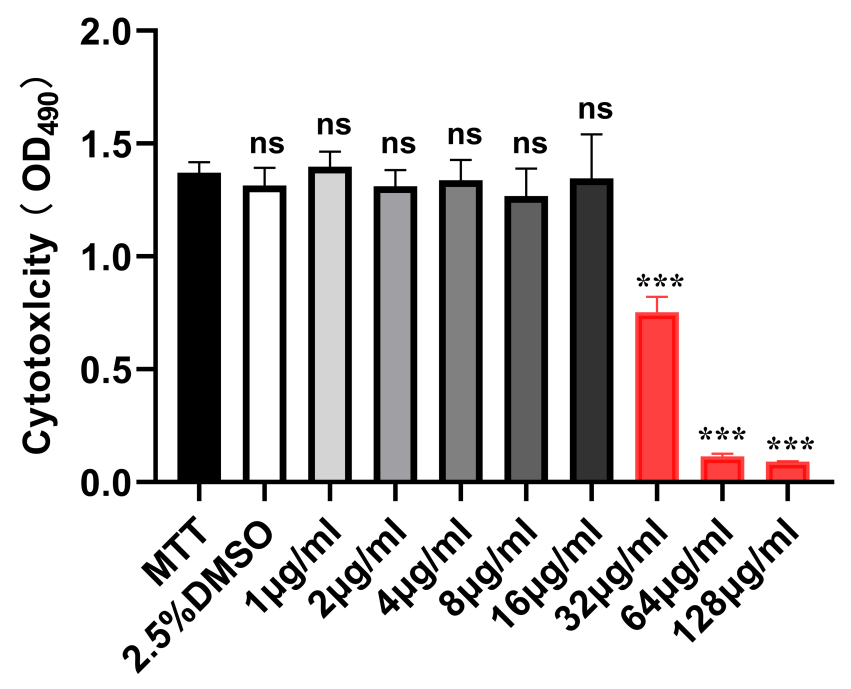

PFK-158 Concentration $(\mu \mathrm{g} / \mathrm{ml})$

Figure 5 Cytotoxic effect of PFK-I58 with different concentrations and $2.5 \%$ DMSO against RAW 264.7 murine macrophage cell line (absorbance values at 490 $\mathrm{nm})$. $* * * p<0.001$. (determined by a two-sample $t$-test). The results are shown as the mean and standard deviation of three independent experiments.

Abbreviations: ns, not significant; DMSO, dimethyl sulfoxide.

PFK-158 against Enterobacteriaceae but also nonfermenting bacteria ( $P$. aeruginosa, $A$. baumannii). In other words, the combination of colistin and PFK-158 showed a wide range of synergistic activity on colistinresistant GNB. This suggests that the mechanism of the synergistic activity of the two drugs combination is probably not related to the difference of the strains property.

Moreover, in order to further explore the reasons for the synergistic effect of colistin and PFK-158, the biofilm matrices and the number of cell morphology were observed by crystal violet staining method and scanning electron microscopy. It showed that colistin combined with PFK-158 can effectively suppress the formation of biofilm and reduce the cell arrangement density of biofilm against all the colistin-resistant $P$. aeruginosa, A. baumannii, $K$. pneumoniae, and half of colistin-resistant $E$. coli that we tested. Based on the results as described above, we speculate that the synergistic effect of colistin and PFK158 may be achieved by inhibiting the formation of biofilm. However, whether the combined application of colistin and PFK-158 will affect the expression of colistin resistance genes and lipid A modification is still worthy of further investigation by us.

Besides, PFK-158 shows extensive anti-tumor activity by reducing the uptake of glucose in cancer cells, the production of ATP, the release of lactic acid and inducing apoptosis and autophagy. ${ }^{15,16}$ And in order to assess the safety of PFK-158, we conducted the cytotoxicity assay using the MTT method. The result illustrated that the PFK$158(<32 \mu \mathrm{g} / \mathrm{mL})$ and $2.5 \%$ DMSO exerted negligible cytotoxicity on the RAW 264.7 macrophage cell lines, which indicates that PFK-158 has good safety and is worthy of further exploration and research.

Finally, it is crucial to evaluate the clinical significance of these observations and to better understand the doseresponse relationships of a combination of colistin with PFK-158. As we all know, colistin has the nephrotoxicity and neurotoxicity ${ }^{41}$ thus, if the combination therapy can lower the concentration and dosage of colistin, it will be extremely meaningful for the clinical treatment of colistinresistant GNB infections, especially for the patients with cancer, who are a special group with the low immunity and are more prone to be infected. Therefore, further clinical study is recommended to assess the potential application of the combination of the two drugs in clinical practice.

\section{Conclusion}

In conclusion, our results strongly exhibited that the combination of colistin and PFK-158 displayed the significantly synergistic activity against all tested colistinresistant GNB. PFK-158 was found to potentiate the antibacterial activity of colistin against a wide panel of colistin-resistant GNB no matter what species (including nonfermenting bacteria and Enterobacteriaceae). Besides, the study explored the antibiofilm potential of colistin combined with PFK-158 against colistin-resistant GNB, which may be the main mechanism of the synergistic effect of the combination of colistin and PFK-158. It may be a potential new alternative as a new antibiofilm group for the treatment of infections caused by the colistin-resistant clinical GNB.

\section{Ethical Statement}

Whole investigation protocols in this study were approved by the Ethics Committee of the First Affiliated Hospital of Wenzhou Medical University. There are no studies with humans or animals performed by any of the authors in this article. Informed consent was waived because this study, with its observational nature, mainly focused on bacteria and gave no interventions to patients.

\section{Acknowledgment}

We thank the Planned Science and Technology Project of Wenzhou (no. Y20170204) for providing financial funding. 


\section{Author Contributions}

All authors made a significant contribution to the work reported, whether that is in the conception, study design, execution, acquisition of data, analysis and interpretation, or in all these areas; took part in drafting, revising or critically reviewing the article; gave final approval for the version to be published; have agreed on the journal to which the article has been submitted; and agreed to be accountable for all aspects of the work.

\section{Disclosure}

The authors report no conflicts of interest in this work.

\section{References}

1. Luepke KH, Mohr JF. The antibiotic pipeline: reviving research and development and speeding drugs to market. Expert Rev Anti Infect Ther. 2017;15:425-433. doi:10.1080/14787210.2017.1308251

2. Falagas ME, Mavroudis AD, Vardakas KZ. The antibiotic pipeline for multi-drug resistant gram negative bacteria: what can we expect? Expert Rev Anti Infect Ther. 2016;14(8):747-763. doi:10.1080/ 14787210.2016.1204911

3. Zhang N, Liu W, Qian K. In-vitro antibacterial effect of tea polyphenols combined with common antibiotics on multidrug-resistant Klebsiella pneumoniae. Minerva Med. 2020;111(6):536-543. doi:10.23736/S0026-4806.20.06459-9

4. MacVane SH. Antimicrobial resistance in the intensive care unit: a focus on gram-negative bacterial infections. $J$ Intensive Care Med. 2017;32(1):25-37. doi:10.1177/0885066615619895

5. Prabaker K, Weinstein RA. Trends in antimicrobial resistance in intensive care units in the United States. Curr Opin Crit Care. 2011;17:472-479. doi:10.1097/MCC.0b013e32834a4b03

6. Abd El-Baky RM, Masoud SM, Mohamed DS, et al. Prevalence and some possible mechanisms of colistin resistance among multidrug-resistant and extensively drug-resistant Pseudomonas aeruginosa. Infect Drug Resist. 2020;13:323-332. doi:10.2147/IDR. S238811

7. Mavroidi A, Likousi S, Palla E, et al. Molecular identification of tigecycline-and colistin-resistant carbapenemase-producing Acinetobacter baumannii from a Greek hospital from 2011 to 2013. J Med Microbiol. 2015;64:993-997. doi:10.1099/jmm.0.000127

8. Parisi SG, Bartolini A, Santacatterina E, et al. Prevalence of Klebsiella pneumoniae strains producing carbapenemases and increase of resistance to colistin in an Italian teaching hospital from January 2012 to December 2014. BMC Infect Dis. 2015;15:244. doi:10.1186/s12879-015-0996-7

9. Olaitan AO, Diene SM, Kempf M, et al. Worldwide emergence of colistin resistance in Klebsiella pneumoniae from healthy humans and patients in Lao PDR, Thailand, Israel, Nigeria and France owing to inactivation of the $\mathrm{PhoP} / \mathrm{PhoQ}$ regulator mgrB: an epidemiological and molecular study. Int $J$ Antimicrob Agents. 2014;44:500-507. doi:10.1016/j.ijantimicag.2014.07.020

10. Çağlan E, Nigiz Ş, Sancak B, et al. Resistance and heteroresistance to colistin among clinical isolates of Acinetobacter baumannii. Acta Microbiol Immunol Hung. 2020;67(2):107-111. doi:10.1556/ 030.66.2019.021

11. Liu YY, Wang Y, Walsh TR, et al. Emergence of plasmid-mediated colistin resistance mechanism MCR-1 in animals and human beings in China: a microbiological and molecular biological study. Lancet Infect Dis. 2016;16:161-168. doi:10.1016/S1473-3099(15)00424-7
12. Falagas ME, Kasiakou SK, Saravolatz LD. Colistin: the revival of polymyxins for the management of multidrug-resistant gram-negative bacterial infections. Clin Infect Dis. 2005;40(9):1333-1341. doi: $10.1086 / 429323$

13. Wijesinghe GK, Feiria SB, Maia FC, et al. In-vitro antibacterial and antibiofilm activity of Cinnamomum verum leaf oil against Pseudomonas aeruginosa, Staphylococcus aureus and Klebsiella pneumoniae. An Acad Bras Cienc. 2021;93(1):e20201507. doi:10.1590/0001-3765202120201507

14. Zhang J, Liang X, Zhang S, et al. Maipomycin A, a novel natural compound with promising anti-biofilm activity against gram-negative pathogenic bacteria. Front Microbiol. 2021;11:598024. doi:10.3389/ fmicb.2020.598024

15. Longo F, Vuotto C, Donelli G. Biofilm formation in Acinetobacter baumannii. New Microbiol. 2014;37:119-127.

16. Yeo HJ, Yoon SH, Lee SE. Bacterial biofilms on extracorporeal membrane oxygenation catheters. ASAIO J. 2018;64(4):e48-e54. doi:10.1097/MAT.0000000000000750

17. Bjarnsholt T. The role of bacterial biofilms in chronic infections. APMIS Suppl. 2013;121:1-51.

18. Chiang WC, Nilsson M, Jensen P. Extracellular DNA shields against aminoglycosides in Pseudomonas aeruginosa biofilms. Antimicrob Agents Chemother. 2013;57:2352-2361. doi:10.1128/AAC.00001-13

19. Mellbye B, Schuster M. The sociomicrobiology of antivirulence drug resistance: a proof of concept. Mbio. 2011;2:119. doi:10.1128/ mBio.00131-11

20. Boisvert AA, Cheng MP, Sheppard DC. Microbial biofilms in pulmonary and critical care diseases. Ann Am Thorac Soc. 2016;13:1615-1623. doi:10.1513/AnnalsATS.201603-194FR

21. Tanner WD, Atkinson RM, Goel RK. Horizontal transfer of the blaNDM-1 gene to Pseudomonas aeruginosa and Acinetobacter baumannii in biofilms. FEMS Microbiol Lett. 2017;364:1-6. doi:10.1093/femsle/fnx048

22. Mondal S, Roy D, Bhattacharya S, et al. Therapeutic targeting of PFKFB3 with a novel glycolytic inhibitor PFK158 promotes lipophagy and chemosensitivity in gynecologic cancers. Int $J$ Cancer. 2019;144:178-189. doi:10.1002/ijc.31868

23. Gustafsson NMS, Färnegårdh K, Bonagas N, et al. T. Targeting PFKFB3 radiosensitizes cancer cells and suppresses homologous recombination. Nat Commun. 2018;9:3872. doi:10.1038/s41467-018-06287-x

24. Zhang Y, Wang X, Li X, et al. Synergistic effect of colistin combined with PFK-158 against colistin-resistant Enterobacteriaceae. Antimicrob Agents Chemother. 2019;63:e00271-19. doi:10.1128/AAC.00271-19

25. Magiorakos AP, Srinivasan A, Carey RB, et al. Multidrug-resistant, extensively drug-resistant and pandrug-resistant bacteria: an international expert proposal for interim standard definitions for acquired resistance. Clin Microbiol Infect. 2012;18:268-281. doi:10.1111/ j.1469-0691.2011.03570.x

26. Stokes JM, MacNair CR, Ilyas B, et al. Pentamidine sensitizes gram-negative pathogens to antibiotics and overcomes acquired colistin resistance. Nat Microbiol. 2017;2:17028. doi:10.1038/ nmicrobiol.2017.28

27. Berard X, Stecken L, Pinaquy JB, et al. Comparison of the antimicrobial properties of silver impregnated vascular grafts with and without triclosan. Eur J Vasc Endovasc Surg. 2016;51:285-292. doi:10.1016/j.ejvs.2015.10.016

28. Zakuan ZD, Suresh K. Rational use of intravenous polymyxin B and colistin: a review. Med J Malaysia. 2018;73:351-359.

29. O'Loughlin CT, Miller LC, Siryaporn A, et al. A quorum-sensing inhibitor blocks Pseudomonas aeruginosa virulence and biofilm formation. Proc Natl Acad Sci USA. 2013;110(44):17981-17986. doi:10.1073/pnas.1316981110

30. Li Y, Huang J, Li L, Liu L. Synergistic activity of berberine with azithromycin against Pseudomonas Aeruginosa isolated from patients with cystic fibrosis of lung in vitro and in vivo. Cell Physiol Biochem. 2017;42(4):1657-1669. doi:10.1159/000479411 
31. Das MC, Sandhu P, Gupta P, et al. Attenuation of Pseudomonas aeruginosa biofilm formation by Vitexin: a combinatorial study with azithromycin and gentamicin. Sci Rep. 2016;6(1):23347. doi:10.1038/srep23347

32. Poirel L, Jayol A, Nordmann P. Polymyxins: antibacterial activity, susceptibility testing, and resistance mechanisms encoded by plasmids or chromosomes. Clin Microbiol Rev. 2017;30(2):557-596. doi:10.1128/CMR.00064-16

33. Dharani S, Kim DH, Shanks RMQ, et al. Susceptibility of colistin-resistant pathogens to predatory bacteria. Res Microbiol. 2018;169(1):52-55. doi:10.1016/j.resmic.2017.09.001

34. Ozger HS, Cuhadar T, Yildiz SS, et al. In vitro activity of eravacycline in combination with colistin against carbapenem-resistant A. baumannii isolates. J Antibiot. 2019;72(8):600-604. doi:10.1038/ s41429-019-0188-6

35. Falagas ME, Voulgaris GL, Tryfinopoulou K, et al. Synergistic activity of colistin with azidothymidine against colistin-resistant Klebsiella pneumoniae clinical isolates collected from inpatients in Greek hospitals. Int $J$ Antimicrob Agents. 2019;53(6):855-858. doi:10.1016/j.ijantimicag.2019.02.021

36. Vidaillac C, Benichou L, Duval RE. In vitro synergy of colistin combinations against colistin-resistant Acinetobacter baumannii, Pseudomonas aeruginosa, and Klebsiella pneumoniae isolates. Antimicrob Agents Chemother. 2012;56(9):4856-4861. doi:10.1128/ AAC.05996-11
37. Bidaud AL, Djenontin E, Botterel F, et al. Colistin interacts synergistically with echinocandins against Candida auris. Int J Antimicrob Agents. 2020;55(3):105901. doi:10.1016/j.ijantimicag.2020.105901

38. Domalaon R, De Silva PM, Kumar A, et al. The anthelmintic drug niclosamide synergizes with colistin and reverses colistin resistance in gram-negative bacilli. Antimicrob Agents Chemother. 2019;63(4): e02574-18. doi:10.1128/AAC.02574-18

39. Cannatelli A, Principato S, Colavecchio OL, et al. Synergistic activity of colistin in combination with resveratrol against colistin-resistant gram-negative pathogens. Front Microbiol. 2018;9:1808. doi:10.3389/fmicb.2018.01808

40. Pollini S, Boncompagni S, Maggio D, et al. In vitro synergism of colistin in combination with $\mathrm{N}$-acetylcysteine against Acinetobacter baumannii grown in planktonic phase and in biofilms. J Antimicrob Chemother. 2018;73:2388-2395. doi:10.1093/jac/dky185

41. Inci A, Toker MK, Bicer IG, et al. Determination of colistin-related nephrotoxicity and risk factors in intensive care unit. North Clin Istanb. 2018;5:120-124. doi:10.14744/nci.2017.42243
Infection and Drug Resistance

\section{Publish your work in this journal}

Infection and Drug Resistance is an international, peer-reviewed openaccess journal that focuses on the optimal treatment of infection (bacterial, fungal and viral) and the development and institution of preventive strategies to minimize the development and spread of resistance. The journal is specifically concerned with the epidemiology of

\section{Dovepress}

antibiotic resistance and the mechanisms of resistance development and diffusion in both hospitals and the community. The manuscript management system is completely online and includes a very quick and fair peerreview system, which is all easy to use. Visit http://www.dovepress.com/ testimonials.php to read real quotes from published authors. 